\title{
Myrtia, $\mathrm{n}^{\circ} 35$ (2020), 451-458
}

\section{L'Odissea e Margaret Atwood \\ The Odissey and Margaret Atwood}

\author{
Federica Boero* \\ Università degli Studi di Genova
}

La scrittrice canadese Margaret Atwood pubblica nel 2005 Il canto di Penelope (The Penelopiad), un romanzo che rilegge l'Odissea di Omero dal punto di vista della sposa di Odisseo, immaginandola confinata ormai da millenni nell'Oltretomba e intenzionata a far conoscere, infine, la sua verità sui fatti di Itaca. Fa da contrappunto al monologo il coro delle dodici ancelle, che il re ha ordinato a Telemaco di uccidere, poiché accusate di aver intrattenuto rapporti illeciti con i Proci. ${ }^{1}$ Il personaggio dell'ancella, una donna oppressa e schiava, fatta oggetto di prevaricazioni disumane da parte del genere maschile, è presente, già nel 1985, nel romanzo che ha reso celebre la scrittrice, Il racconto dell'Ancella (The Handmaid's Tale $)^{2}$, oggi assunto a simbolo delle rivendicazioni del movimento $M e$ too per il

Dirección para correspondencia: Dipartimento di Antichità, Filosofia, Storia (DAFIST), via Balbi 4, Università degli Studi di Genova- IT- 16126 Genova. E-mail: federicaboero@hotmail.it

${ }^{1}$ V. Omero, Odissea XXII, v. 440 ss.

2 Il racconto dell'ancella, pubblicato nel 1985, è un romanzo fantascientifico ambientato in un futuro post-apocalittico, dove l'inquinamento, i cambiamenti climatici e una serie di disastri nelle centrali nucleari californiane rischiano di provocare l'estinzione del genere umano. Le donne hanno difficoltà a procreare e spesso danno alla luce creature deformi o destinate a una morte precoce. Per questo, il regime monoteocratico di Gilead, che con un colpo di Stato si è insediato al governo degli Stati Uniti, attua un piano finalizzato a privare le donne della loro libertà personale, proibendo che lavorino o che possiedano un conto in banca, e costringendo quelle ancora in età fertile a generare figli per l'élite dominante. Esse prendono il nome di "ancelle", vestono tuniche rosse e coprenti, e intrattengono rapporti sessuali con i Comandanti in presenza delle loro mogli legittime. Nel caso in cui non riescano a mettere al mondo un figlio, vengono considerate "Nondonne" e deportate nelle Colonie. La voce narrante del romanzo è Offred, l'ancella del Comandante Fred. Strappata dalla figlia e dal compagno Luke, Offred svolge le proprie mansioni con rassegnazione, costretta a partecipare a riti inconcepibili e a pratiche disumane. Tutto cambia, però, quando un'altra ancella la mette a parte dell'esistenza di un movimento segreto, il Mayday, determinato a cambiare la situazione, e le chiede di raccogliere informazioni sul Comandante Fred. Spaventata, Offred non contribuisce alle ricerche e continua a onorare i propri doveri per sopravvivere, cedendo alle richieste del Comandante, 
suo messaggio fortemente femminista. ${ }^{3}$ In questo articolo si intende dimostrare che proprio ne II racconto dell'Ancella va ricercato lo spunto, che ispirò all'autrice una rivisitazione omerica in cui le serve della reggia di Itaca sono coprotagoniste di Penelope, nell'azione e nelle sofferenze patite. Sebbene l'ambientazione e il genere siano differenti, medesimo è lo spirito che anima le due vicende e che trova concreto sviluppo nel personaggio simbolo dell'ancella.

Il canto di Penelope è un lungo racconto-confessione, costruito all'interno di una cornice ambientata nell'Oltretomba, negli anni Duemila. Il testo è strutturato in modo da richiamare l'alternarsi degli episodi e dei canti corali della tragedia classica; le parole della regina, che espone gli avvenimenti che hanno caratterizzato la sua vita, si alternano, infatti, agli interventi del coro delle ancelle uccise, recitati in forme diverse a seconda dell'attinenza con l'argomento trattato. ${ }^{4}$

Il primo intervento del coro è una filastrocca, che l'autrice immagina accompagnare il gioco del salto con la corda. Sarcasticamente, l'argomento del brano è l'impiccagione delle ancelle:

con cui inizia a incontrarsi anche di nascosto dalla moglie. Quest'ultima le chiede di farsi mettere incinta da Nick, l'autista, in quanto consapevole della sterilità del marito. In cambio, le promette notizie della figlia scomparsa. Offred e Nick iniziano una relazione clandestina e tra loro sembra nascere un'intesa, ma, quando la moglie del Comandante scopre le attenzioni non consentite che il marito ha rivolto all'ancella, l'equilibrio si spezza. Terrorizzata dalle minacce della donna e venuta a conoscenza del suicidio di un'altra ancella coinvolta nel movimento di ribellione, Offred si appresta a togliersi la vita, ma è raggiunta da Nick, che la informa che è arrivato il Mayday, il giorno del suo salvataggio ad opera dei ribelli. Nel finale, Offred viene prelevata e caricata sul furgone degli "Occhi” (i Servizi segreti del governo), sotto lo sguardo del Comandante e della moglie. Mentre sale sul furgone, l'ancella ancora non sa se si tratti davvero del giorno della sua fuga o di quello della sua cattura.

${ }^{3}$ Oggi i movimenti femministi identificano nell'ancella il simbolo delle rivendicazioni del genere femminile nei confronti dell'uomo e leggono ne Il racconto dell'ancella un archetipo letterario delle rimostranze che ispirano la loro lotta. In realtà, nel 1985, il romanzo non ebbe un grande successo di pubblico, ma fu celebrato soltanto marginalmente dalla critica. Nel 1990, ispirò un primo adattamento per lo schermo. Nel cast, figuravano attori come Faye Dunaway, Robert Duvall, Natasha Richardson e Aidan Quinn, tuttavia fu un flop al botteghino. Solo recentemente, con l'elezione di Donald Trump e le sue clamorose dichiarazioni di stampo sessista, il romanzo venne riscoperto dalla piattaforma Hulu, che ne fece una serie di successo nel 2017, arrivata oggi alla quarta stagione.

${ }^{4}$ Se per celebrare la nascita di Telemaco le ancelle recitano un idillio, i viaggi di Odisseo sono ricordati con un canto marinaresco e ai tradimenti di Penelope è riservato un breve spettacolo teatrale in rima. 


Siamo le ancelle
ci hai condannate
ci hai ammazzate
nell'aria appese
lo spasmo ai piedi
non era giusto
regine, dee
o prostitute
tutte le hai godute
cos'abbiam fatto
rispetto a te
che ci hai dannate.

Le ancelle, nell'Oltretomba, si rivolgono a Odisseo e chiedono il perché della loro condanna. Sono state impiccate, la stessa pena scelta per loro da Telemaco nell'Odissea (XXII, vv. 465-473). L'impiccagione, come strumento di repressione e di monito per chi resta, incombe quasi ossessivamente nel Racconto dell'ancella, dove i dissidenti al regime di Gilead vengono impiccati ed esposti presso un apposito «muro", visibile alla cittadinanza. Le ancelle uccise istituiscono un impossibile parallelo tra la propria condizione e quella di Odisseo: se la loro colpa è stata quella di avere rapporti sessuali con i Proci, ben più grave giudicano la condotta del re, che ha tradito la moglie in numerose occasioni e ha l'ardire di ergersi a giudice nei loro riguardi. Nel Racconto dell'ancella, questa dicotomia è rintracciabile nel comportamento del Comandante Fred, rappresentante della casta che ha condannato le donne statunitensi alla segregazione e alla schiavitù, per colpa dei loro costumi giudicati troppo liberi, e che, nel corso della vicenda, si rivela essere frequentatore di un locale a luci rosse, in cui i Comandanti si concedono piaceri proibiti, di nascosto dalle legittime consorti.

Il racconto di Penelope prende le mosse dall'infanzia della regina e dal suo difficile rapporto con i genitori ${ }^{6}$, soffermandosi poi sui sentimenti contrastanti che prova nei confronti di Odisseo, che la ottiene con l'inganno, barando a una gara di corsa contro gli altri pretendenti. Odisseo la porta a Itaca e, sebbene Penelope non si trovi a suo agio tra la suocera Anticlea e l'invadente nutrice Euriclea, finisce per

${ }^{5}$ M. Atwood, 2018, p. 15.

${ }^{6}$ Il padre, il re Icario, tenta di annegarla per difendersi da un vaticinio, facendole perdere completamente la fiducia in lui, mentre la madre, una nereide, è assente e del tutto anaffettiva. 
innamorarsi dello sposo, che sa conquistarla con le sue doti di narratore. Presso la reggia, viene introdotta la figura di un'ancella, Attoride, regalata dal re Icario alla figlia per le nozze: «Era tutt'altro che felice di trovarsi a Itaca. Non avrebbe voluto lasciare la ricchezza del palazzo di Sparta e le amiche che aveva tra la servitù». ${ }^{7}$ Uno dei suoi compiti è restare tutta la notte di sentinella, davanti alla porta della camera da letto dei regnanti, affinché nessuno li disturbi. Penelope sottolinea che il padre ha scelto una donna non più giovane, né avvenente, in modo che non sia oggetto di interesse da parte di Odisseo. Si ripropone, così, il ruolo di una donna che interviene nelle dinamiche della coppia, coinvolta in prima persona nella loro sfera privata, eppure spogliata della propria femminilita al punto, da non costituire una minaccia per la stabilità dei coniugi. Questo stesso rapporto è ravvisabile nel "triangolo", che nel regime di Gilead lega i Comandanti e le mogli all'ancella loro affidata, preposta alla funzione procreativa: le ancelle hanno rapporti sessuali con i Comandanti sotto gli occhi delle mogli, ma ciò non costituisce un tradimento, proprio perché esse sono private della propria individualità e assumono la valenza di un mezzo, di una «necessità $^{8}$ per garantire una discendenza alla famiglia. Per annullarne completamente la femminilità dal punto di vista fisico, la legge impone che vestano tuniche rosse, larghe e coprenti, e che portino veli sul capo e alette bianche ai lati del viso, in modo da impedire loro di entrare in contatto visivo diretto con gli altri.

Al racconto di Penelope sulla nascita di Telemaco e sui suoi primi giorni di vita, le ancelle rispondono con un altro intervento, definito dall'autrice un "idillio", volto a sottolineare l'ingiustizia della loro condizione, l'incomprensibile gioco del destino che volle concedere loro una vita da schiave e a Telemaco il titolo di principe:

Le nostre vite intrecciate alla sua, bambine quando lui era bambino, cuccioli, giochi, finte sorelline, crescevamo, minuscole compagne.

E quando lui correva, noi correvamo

Sempre più sporche e più affamate, con più macchie di sole e il piatto vuoto.

Ma gli appartenevamo di diritto, per lavarlo, nutrirlo e divertirlo, poi, incerte barchette di noi stesse, cullarlo fino al sonno.

\footnotetext{
${ }^{7}$ M. Atwood, 2018, p. 50.

${ }^{8}$ M. Atwood, 2004, p. 25.
} 
Non sapevamo, allora, sulle rive della pietrosa isoletta di capre, che lui un giorno, con occhi di gelo, quasi un ragazzo, ci avrebbe impiccate. Saremmo mai riuscite ad affogarlo? Tanto i bambini non hanno morale. ${ }^{9}$

Le ancelle lamentano il fatto di appartenere a Telemaco che, ancora ragazzo, ha il diritto di ucciderle senza subire le conseguenze del suo gesto. Sembrerebbe trattarsi di una distanza, motivata dal diverso status sociale delle schiave rispetto al padrone, ma a Margaret Atwood preme soprattutto insistere sulla contrapposizione tra il coro e Telemaco, in quanto uomo. Non ci sono corrispettivi maschili delle ancelle alla reggia di Odisseo. Gli uomini che lavorano alle dipendenze del re, come allevatori e nei campi, acquisiscono un rispetto dovuto alla fatica e al loro ruolo all'interno dell'economia dell'isola, mentre le schiave nascono già «indifese», destinate ai lavori più umili e a vedere il loro corpo violato impunemente: possono essere concesse dal re agli ospiti graditi, ma vengono accusate di tradimento, qualora siano violentate da un nemico. E' la responsabilità il punto cruciale che segna la distanza tra le ancelle traditrici dell'Odissea e quelle protagoniste del Canto di Penelope, che Margaret Atwood riabilita, descrivendole come alleate della regina, spie che le riferiscono i discorsi dei Proci e che si avvicinano a loro soltanto al fine di esserle d'aiuto, complici nel tessere il sudario di Laerte:

Ad aiutarmi in questo compito laborioso avevo scelto dodici ancelle - le più giovani, perché erano cresciute sotto i miei occhi. Le avevo comprate o me le ero fatte regalare da piccole, come compagne di gioco per Telemaco, e le avevo istruite personalmente affinché imparassero tutto quello che dovevano sapere sulla vita che si svolgeva a palazzo. Erano cordiali, piene di energia, qualche volta parlavano o ridevano troppo forte, come tutte le ancelle più giovani, ma mi rallegrava sentirle chiacchierare o cantare. Avevano imparato a usare bene le loro voci. Erano gli occhi e le orecchie di cui mi potevo fidare a palazzo; per più di tre anni mi aiutarono a disfare la trama, nel cuore della notte, con le porte chiuse, alla luce di una torcia. Dovevamo fare attenzione, parlare sottovoce, eppure sembrava una festa, ridevamo spesso. [...] I «sissignora» e i «nossignora» delle ancelle acquistavano una sottile, ironica gaiezza, come se nessuna di noi credesse all'esistenza di un rapporto servile. ${ }^{10}$

\footnotetext{
${ }^{9}$ M. Atwood, 2018, pp. 58-59.

10 M. Atwood, 2018, p. 90.
} 
L'innocenza oggettiva delle ancelle viene sottolineata, anche quando Penelope parla della scoperta dell'inganno del telaio da parte dei Proci:

Purtroppo un'ancella tradì il segreto di quel mio tessere senza fine. Sono sicura che accadde per errore: le ragazze sono spesso sventate, le dev'essere sfuggito un accenno, una parola. Ancora adesso non so chi fu tra loro a tradirmi. ${ }^{11}$

Le ancelle di Penelope sono vittime di una legge declinata al maschile, che non giudica loro diritto disporre liberamente del proprio corpo. Analogamente, nel Racconto dell'ancella, lo Stato priva di ogni libertà personale le donne statunitensi, impedendo loro di vestirsi in maniera vistosa, di convivere e avere figli al di fuori del matrimonio, di decidere se interrompere una gravidanza indesiderata. Eppure, è lo stesso Comandante a insistere affinché Offred indossi un vestito di lustrini e lo accompagni presso un locale di prostitute, contravvenendo alle regole. Quando la moglie scopre l'abito e comprende che tra il marito e l'ancella si è creato un legame diverso da quello consentito, medita una punizione per lei, pur consapevole che la sua posizione di schiava non le avrebbe dato modo di rispondere con un rifiuto al volere del Comandante. La responsabilità ricade comunque sull'ancella e nessuno prende le sue difese. Si consideri che, nel poema omerico, la strage delle dodici ancelle di Itaca non viene fermata da Penelope: la regina dorme mentre Odisseo le uccide e, pur sapendo di essere stata lei a spingerle tra le braccia dei Proci, anche in seguito preferisce tacere riguardo all'ingiustizia compiuta dal suo sposo, non riscattando nemmeno la loro memoria, nel timore di mettere se stessa in una posizione pericolosa. ${ }^{12}$

Terminato il racconto con il ricongiungimento dei sovrani, la cornice narrativa riporta l'azione al presente, in un'aula di tribunale del ventunesimo secolo, dove Odisseo viene processato per la strage dei Proci: di fronte all'assoluzione del re, le ancelle intervengono esponendo le proprie ragioni. Tuttavia, il giudice scagiona Odisseo anche da questa accusa, dicendo che, in epoca arcaica, il loro assassinio rientrava nei diritti legittimi di un sovrano: le ancelle non sono colpevoli di essersi lasciate stuprare, ma di non aver chiesto prima il permesso al re. La violenza perpetuata ai loro danni non si identifica come un reato, ma viene definita una «deplorevole consuetudine della vita che si svolge a palazzo». ${ }^{13}$ Anche il regime di Gilead ammette la violenza nei confronti delle ancelle ed essa diviene una

${ }^{11}$ M. Atwood, 2018, p. 91.

${ }^{12} \mathrm{Le}$ ancelle, esibendosi in uno «spettacolo teatrale» intitolato «Le peripezie di Penelope», accusano la regina di averle tradite consapevolmente, preoccupata che parlassero delle sue scappatelle con Anfinomo.

${ }^{13}$ M. Atwood, 2018, p. 140. 
consuetudine, tutelata dalla legge. Le donne preposte dal governo alla procreazione meritano quel trattamento, in quanto hanno avuto in precedenza comportamenti giudicati vergognosi.

Alle ancelle, ritenute inderogabilmente colpevoli, non resta che invocare le dee della Vendetta, dodici Erinni, che chiudono il racconto giurando di perseguitare Odisseo per l'eternità:

$$
\begin{aligned}
& \text { Innominate } \\
& \text { vite vendute } \\
& \text { le condannate } \\
& \text { a dir di sì } \\
& \text { ma di quel sì } \\
& \text { ci hanno incolpate } \\
& \text { non era giusto } \\
& \text { però quaggiù } \\
& \text { insieme a noi } \\
& \text { sei anche tu. } \\
& \text { Noi ti cerchiamo } \\
& \text { noi ti seguiamo. }{ }^{14}
\end{aligned}
$$

Unite, le ancelle si ribellano, non con un'azione volta a migliorare la propria condizione, come nel Racconto dell'ancella, ma con un'inedita azione persecutoria nei confronti di chi le ha condannate, mettendo persino in ombra la figura di Penelope, pronta a riprendere nel suo letto l'uomo che l'ha tradita e che ha frustrato, facendo ritorno, ogni sua aspirazione ad occupare un ruolo di potere nella reggia.

Il messaggio che la scrittrice vuole trasmettere ha inevitabilmente un collegamento con l'attualità. Margaret Atwood, da sempre in prima linea nella difesa dei diritti delle donne, contro la violenza di genere, muove una critica aspra verso una società in cui le parole di chi subisce uno stupro possono essere messe in dubbio, in cui spesso il modo di vestire della vittima o un presunto atteggiamento consenziente vengono giudicate attenuanti a favore degli imputati. Seppure i due romanzi siano stati pubblicati nel 1985 e nel 2005, il nuovo interesse nei loro confronti è determinato dalla complessità del personaggio dell'ancella e dalla riflessione sul presente, che è in grado di innescare nel lettore.

${ }^{14}$ M. Atwood, 2018, p. 149. 


\section{Bibliografia}

M. Atwood, 2004, Il racconto dell'ancella, Milano, trad. it. a cura di C. Pennati. M. Atwood, 2018, Il canto di Penelope, Milano, trad. it. a cura di M. Crepax. Omero, 2010, Odissea, Milano, trad. it. a cura di G.A. Privitera. 